\title{
Benefícios de uma dieta quimicamente definida com baixo teor de resíduos para pacientes com tumor de canal anal submetidos a radioquimioterapia associada
}

\author{
The benefits of a chemically defined diet for patients with anal canal \\ tumour submitted to radiotherapy and chemotherapy associated
}

Márcia Henriques Teixeira

\begin{abstract}
Resumo
Pessoas com câncer apresentam freqüentemente desnutrição, o que el eva as taxas de complicações e mortalidade. Tumores do trato digestivo levam a obstruções, as quais interferem na absorção de nutrientes levando à perda ponderal. O s sintomas do câncer de canal anal são dor, sangramentos, diarréia. 0 tratamento radioquimioterápico leva à presença de náuseas, vômitos, diarréia, fibrose do canal anal e radioepidermite. D esta forma, faz-se necessária a utilização de dietas pobres em resíduo a fim de prevenir a impactação fecal, reduzir a freqüência e esforço de evacuações e volume fecal, evitando a piora do quadro. Entretanto, a dieta líquida in natura com baixo teor de resíduos fornece baixo aporte calórico-proteico. Sendo assim, o trabalho objetiva descrever a importância da suplementação alimentar através de uma dieta quimicamente definida pobre em resíduos, na adequação calórica-proteica de pacientes com tumor decanal anal em radioquimioterapia, evitando o agravamento da depleção nutricional. Foi realizado levantamento bibliográfico, sendo consultados livros clássicos, artigos de revistas indexadas brasileiras e internacionais acessados no site da M edline para a obtenção de informações relativas ao tema. O s estudos evidenciam que a suplementação de dietas quimicamente definidas oferece adequação da oferta de nutrientes, boa aceitação, bem-estar emocional e melhora da ingestão alimentar. D esta forma pode-se concluir que a suplementação alimentar está indicada para pacientes que apresentem depleção do estado nutricional, principalmente naqueles em que se faz necessário o uso de dietas restritivas, como a dieta com baixo teor de resíduos, utilizadas por pessoas portadoras de tumor de canal anal.

Palavras-chave: dieta com baixo resíduo; suplementação alimentar; fibra na dieta; neoplasias do ânus; transtornos nutricionais; estado nutricional.
\end{abstract}




\begin{abstract}
$M$ alnutrition is observed in patients with cancer, and this is a factor that increases complications and mortality. $M$ alignant tumours of the digestive tract result in obstructions that interfere in the absorption of nutrients, leading to weight loss. The symptoms of cancer of the anal canal are pain, bleeding, diarrhea. The treatment with radiochemotherapy result in nausea, vomit, diarrhea, fibrosis of the anal canal and radioepidermal inflammation. H ence, it is necessary to prescribe a low-residue diet to prevent fecal impactation, as well as to reduce the frequency and effort of evacuations and fecal volume. However, fluid diets in natura with low-residue rate, provide low calory and protein intake. The aim of this study was to describe the importance of the food supplementation through a chemically low-residue diet, with the adequate provision of calories and protein in patients with cancer of the anal canal submitted to radiochemotherapy, therefore, contributing, to prevent the aggravation of the nutritional depletion. This study consists in a review of classical books, articles from specialized indexed Brazilian internacional journals present in the $M$ edline web site In this study it becomes evident that the nutritional supplementation of chemically-defined diets offers a proper amount of the nutrients, good acceptance, emotional well-being and an increase in food ingestion. H ence, we conclude that food supplements are indicated to patients that present depletion of the nutritional status, especially those to whom the use of restrictive diets is necessary, such as low-residue diet for of the anal canal.
\end{abstract}

Key words: low residue diet; alimentary supplementation; dietary fiber; anus neoplasms; nutrition disorders; supplementary feeding.

\section{INTRODUÇÃO}

No Brasil observa-se uma mudança no perfil epidemiológico caracterizada pela queda progressiva das doenças infecciosas e parasitárias e pelo aumento das doenças crônico-degenerativas, entre elas 0 câncer. ${ }^{1}$

Pacientes portadores de câncer apresentam freqüentemente desnutrição proteicocalórica. ${ }^{2-4} \mathrm{~A}$ alta incidência de desnutrição em pacientes oncológicos, mesmo antes da realização do tratamento para o câncer (cirurgia, quimioterapia e radioterapia) é um fator que acarreta altas taxas de complicações e mortalidade. ${ }^{2,3,5}$

A etiologia da desnutrição no indivíduo com câncer é multifatorial. ${ }^{2,4-6}$ Pode estar relacionada a diversas substâncias anorexígenas produzidas pelo tumor ou hospedeiro, à presença de dor e obstrução do trato gastrointestinal. ${ }^{5}$

Lesões malignas localizadas ao longo do trato digestivo, entre elas o tumor de canal anal, levam a obstrução parcial ou total de um ou mais sítios, interferindo na absorção de nutrientes e induzindo a perda de peso severa no indivíduo. ${ }^{3,7}$
0 uso de dietas com baixo teor de resíduos faz-se necessário a fim de que se produza pouco resíduo fecal, com o objetivo de evitar a retenção de fezes no trato digestivo estenosado, reduzir a freqüência das evacuações e com isso a dor, sangramentos e irritação local durante o tratamento. ${ }^{7}$

A dieta líquida com baixo teor de resíduos obtida a partir de alimentos in natura apresenta um aporte calórico e proteico deficiente. ${ }^{8} 0$ uso da suplementação alimentar industrializada está indicada para aumentar a ingestão de nutrientes quando não se consegue atingir as necessidades calórico-proteicas através da dieta produzida com alimentos naturais. ${ }^{2}$

D esta forma, este estudo visa a descrever os benefícios da suplementação alimentar oral, através de uma dieta quimicamente definida com baixo teor de resíduos, na adequação calórica e proteica de pacientes com tumor de canal anal submetidos a quimio e radioterapia combinadas.

\section{METODOLOGIA}

Estetrabalho consisteem um levantamento bibliográfico referente ao período de 1987 a 
2000, sendo consultados livros clássicos, artigos de revistas brasileiras e internacionais indexadas e Internet (M edline) para a obtenção de informações relativas ao tema estudado. Foram utilizadas como palavras-chave: dieta com baixo resíduo, suplementação alimentar, câncer de canal anal e desnutrição no câncer.

A síntese dos conteúdos foram descritas na forma de textos para posterior discussão e conclusão dos objetivos.

\section{CAQUEXIA DO CÂNCER}

A caquexia é uma síndrome que indica um estado de perda de tecidos musculares na presença de várias doenças, entre elas o câncer, nas quais a resposta inflamatória do hospedeiro é importante. ${ }^{9}$

Existem duas formas de caquexia: primária e secundária. A primária é o tipo mais comum e surge por interações metabólicas entre o tumor e o hospedeiro. A caquexia secundária ocorre por diminuição na ingestão e absorção de nutrientes por obstruções tumorais do trato gastrointestinal, anorexia por efeito da rádio e quimioterapia, e ressecções intestinais maciças. É comum o encontro simultâneo dos dois tipos de caquexia em um só indivíduo. ${ }^{4,6}$

Dependendo do tipo histológico e do estágio do tumor, as pessoas portadoras de câncer podem ser hipermetabólicas (25\%), normometabólicas (41\%) ou hipometabólicas $(33 \%)$. Alguns tumores, apesar de hipometabólicos, causam perda de peso e caquexia. Isto se deve ao fato de que as alterações do gasto energético parecem não ser 0 principal fator responsável pelo balanço energético negativo, e sim a diminuição da absorção de nutrientes e da ingestão alimentar pelo paciente com câncer. ${ }^{4,6,10}$

\section{CÂNCER DE CANAL ANAL}

0 carcinoma de canal anal é responsável por cerca de $1 \%$ a $4 \%$ dos tumores do intestino grosso, ${ }^{11}$ sendo a principal faixa etária acometida dos 40 a 60 anos. ${ }^{12}$ Predomina no sexo feminino, sendo a proporção de mulheres acometidas em relação aos homens de 2:1 a 4:1.13 Este predomínio no sexo feminino sugere que esteróides sexuais podem direta ou indiretamente ter um lugar em sua gênese. ${ }^{11}$

0 diagnóstico clínico é determinado pela presença de dor associada ou não a sangramentos, sendo estes relatados como um sintoma muito freqüente, ${ }^{11}$ irritação local, tenesmo não aliviado com a evacuação, obstrução e aumento dos linfonodos inguinais nos casos avançados. ${ }^{13}$

Em estudo realizado por Corrêa, ${ }^{14}$ observou-se que os sintomas mais freqüentes apresentados pelos pacientes com este tipo de tumor foram sangramento, presença de tumor, dor, secreção anal, diarréia e eliminação de fezes em forma de fita.

Em 1974, foi proposta a associação de quimioterapia e radioterapia como alternativa terapêutica para este carcinoma. ${ }^{14}$ O s seus resultados mostraram bons resultados com redução dos efeitos actínicos, resultantes da radioterapia exclusiva. ${ }^{14}$

A radioterapia é mais útil como uma forma detratamento local do tumor, enquanto a quimioterapia é usada para controlar a doença metastática clínica ou microscópica. ${ }^{15,16}$

O s efeitos da combinação QT e RT podem ser descritos como independentes, aditivos ou interativos. Estetipo detratamento pode ser administrado seqüencialmente ou simultaneamente. ${ }^{17}$

A toxicidade da combinação QT / RT requer atenção já que o dano causado aos tecidos normais está, em geral, aumentado nesta modalidade de tratamento. ${ }^{15}$

Em estudo realizado por Corrêa ${ }^{14}$ com pacientes com tumor de canal anal, a ocorrência de radioepidermite, náusea, diarréia, fibrose do canal anal e vômito foram as complicações mais freqüentes. Estas atuam influenciando diretamente na diminuição da ingestão alimentar nestes indivíduos, o que mostra o efeito da terapia antitumoral como mais um fator que contribui para a perda ponderal nestes indivíduos.

D esta forma, a dietoterapia mostra-se como um elemento importante a fim de evitar o agravamento da depleção nutricional presente nestes pacientes. 


\section{TRATAMENTO DIETOTERÁPICO NO TUMOR DE CANAL ANAL}

D urante o tratamento deste tumor é indicado o uso de dietas com baixo teor de resíduos, de consistência e volume adaptados ao estadiamento da doença do paciente, que entretanto é nutricionalmente inadequada por fornecer baixo aporte calórico e proteico. ${ }^{8,18}$ A dieta líquida artesanal com alimentos in natura com baixo teor de resíduo apresenta, em média, de 700 a $900 \mathrm{~K} \mathrm{cal} / \mathrm{dia}$ e de 20 a 30 gramas de proteína/dia, ${ }^{19}$ o que fica aquém das recomendações nutricionais preconizadas pela RDA/1989 para a média de um indivíduo adulto.

0 uso de fórmulas quimicamente definidas com baixo teor de resíduo e com aporte calórico e proteico que complemente a dieta com pouco resíduo artesanal (in natura) parece estar mais indicada para evitar o agravamento da depleção nutricional em pacientes com tumor de canal anal tratados com radioquimioterapia associada. ${ }^{8}$

Existem no mercado nacional fórmulas quimicamente definidas com baixo teor de resíduos que podem ser utilizadas como complementação alimentar oral para estes pacientes, oferecendo valores calóricos e proteicos maiores quando utilizados conjuntamente com as dietas com baixo resíduo convencionais, já que apresentam boa aceitação pelos pacientes pela maior variedade de sabores. ${ }^{18}$

\section{DIETAS COM BAIXO TEOR DE RESÍDUOS}

A restrição de resíduo e de fibra na dieta são utilizados para prevenir a impactação de fezes no trato digestivo estenosado e reduzir a freqüência e volume fecal, enquanto prolonga o tempo de trânsito intestinal. ${ }^{7}$

U ma dieta com baix xa fibra não é sinônimo de dieta com pouco resíduo. Fibra é a porção de carboidratos inpossíveis de serem digeridas pelas enzimas do trato gastrointestinal humano, contribuindo para aumentar 0 bolo fecal. Resíduos são elementos dietéticos que não são absorvidos e ficam na luz intestinal após a digestão, contribuindo para aumentar o bolo fecal e aumentar o seu peso apesar de seu baixo conteúdo em fibras. ${ }^{7} 0$ resíduo inclui fibra dietética e também outros constituintes dietéticos, dentre eles os minerais, principalmente cálcio e ferro, açúcares não digeridos, especialmente a lactose e carnes com cartilagem, células gastrointestinais de revestimento e as bactérias intestinais. $^{8}$

D esta forma, a dieta de resíduo mínimo é aquela onde estão excluídos os alimentos com teor moderado a alto de fibras, como 0 leite e derivados e carnes com tecido conjuntivo. ${ }^{8}$ Esta dieta é nutricionalmente inadequada e deve ser usada apenas por curto período de tempo. Sem um planejamento cuidadoso, este tipo de dieta pode levar à desnutrição. ${ }^{8}$

\section{SUPLEMENTAÇ̃̃O ALIMENTAR}

A administração oral de suplementos nutricionais industrializados é indicada quando o paciente é incapaz de ingerir suas necessidades calórico-proteicas através da dieta oral constituída de alimentos naturais. ${ }^{2}$

A suplementação alimentar pode reverter a desnutrição proteico-energética, restaurando a função imunológica e melhorando a tolerância ao tratamento em alguns tipos de tumores. ${ }^{20}$

O s pacientes devem ser estimulados a receber dietas via oral, pois é uma forma fisiológica de alimentá-los e estimulá-los a contribuir para o seu tratamento, exercendo um efeito psicológico muito positivo. ${ }^{21} \mathrm{~A}$ suplementação alimentar é o mais simples e menos agressivo método de aumentar a ingestão de nutrientes. ${ }^{10} \mathrm{Com} 0$ avanço da farmacotécnica, têm surgido suplementos orais cada vez mais valiosos do ponto de vista nutricional, com sabores atraentes e bem aceitos. ${ }^{22}$ Alguns suplementos comerciais são completos e incluem todas as vitaminas e minerais necessários, ao passo que outros se restringem às calorias e proteínas. ${ }^{22}$

Para o sucesso desta terapia é imprescindível que seja feito um trabalho educativo com o paciente, informando-o sobre 0 valor nutricional do suplemento, pois assim ele é motivado. ${ }^{22}$

0 uso da suplementação alimentar em pacientes oncológicos mostrou ganho ponderal, balanço nitrogenado positivo, melhora da função imunológica e do bemestar geral, tanto físico quanto psicológico, 0 que sugere que a resposta à terapia pode estar 
favorecida se o paciente estiver bem nutrido. ${ }^{10}$ M cwhirter e Pennington ${ }^{23}$ relatam que pacientes com câncer demonstraram uma redução na perda de peso e sensação de bemestar após uso de suplementação alimentar.

Em estudo realizado por $\mathrm{N}$ ayel et $\mathrm{al}^{20} \mathrm{com}$ pacientes com câncer de cabeça e pescoço submetidos à radioterapia, foi verificado que todos os pacientes que receberam suplementação alimentar apresentaram ganho ponderal, aumento na dobra cutânea triciptal e na circunferência média de braço, além de terem realizado a radioterapia sem interrupção, enquanto que o grupo que não recebeu suplementação apresentou perda ponderal, diminuição de circunferência média de braço e de dobra cutânea triciptal, além de que $42 \%$ teve suspensa a radioterapia devido a severa mucosite ou precária condição clínica. Os pacientes que receberam suplementação alimentar apresentaram mucosite menos intensa e de menor duração do que o grupo controle. I sto pode ser atribuído à melhor função imunológica dos pacientes suplementados, o que preveniu infecção secundária na mucosa irradiada.

Trabalho realizado com pacientes desnutridos hospitalizados evidenciou que $64 \%$ daqueles que receberam suplementação alimentar apresentaram ganho ponderal, enquanto que $73 \%$ do grupo controle apresentaram perda de peso, mostrando que aqueles que receberam suplementação nutricional durante a hospitalização aumentaram significativamente a ingestão de nutrientes e apresentaram ganho de peso, evidenciando que a suplementação alimentar oral deve ser utilizada como tratamento de escolha para pacientes desnutridos. ${ }^{23}$

A suplementação líquida quimicamente definida é freqüentemente oferecida a pacientes com câncer que apresentam hiporexia, saciedade precoce e perda de peso. ${ }^{24}$ Alguns autores mostram resultados controversos e afirmam que o uso da suplementação oral entre as refeições pode levar a redução de ingestão alimentar pela substituição de alimentos sólidos por estes, uma vez que o líquido é mais fácil de ingerir e engolir. ${ }^{25}$ Tal questão é discutida por $\mathrm{M} \mathrm{CC}$ arthy e Weihofen ${ }^{25}$ que observaram que em pacientes submetidos à radioterapia os suplementos alimentares podem ser usados para aumentar a ingestão calórica e proteica e que não levaram à redução da ingestão alimentar em relação ao grupo controle. ${ }^{25}$
Os achados supra citados são corroborados por estudos como de 0 vesen $e$ Allingstrup, ${ }^{24}$ que evidenciaram que em pacientes oncológicos que receberam suplemento, a progressiva perda ponderal observada no período pré-suplementação não ocorreu nos meses em que foi feita a suplementação.

Q uestiona-se o uso diário e contínuo do suplemento líquido provocando a fadiga do paladar e conseqüentemente um decréscimo na ingestão de muitos pacientes, mas estudos que verificaram a ingestão dos suplementos por período maior que um mês não evidenciaram esteachado como um problema significativo. ${ }^{24}$

Estudo realizado com pacientes que necessitavam de preparo no pré-operatório de cirurgias intestinais mostrou que o grupo que recebeu suplementação com dieta líquida nutricional mente completa com baixo resíduo apresentou limpeza do intestino idêntica a do grupo que utilizou a tradicional dieta líquida com baixo resíduo, sendo que a aceitação da suplementação foi superior à dieta líquida com baixo resíduo. Além disso, a suplementação forneceu significativamente melhor aporte de energia, proteína, vitaminas e minerais. ${ }^{18}$

No grupo que recebeu a suplementação, o balanço de nitrogênio foi significativamente positivo, os valores de albumina sérica foram preservados e houve retorno mais rápido do peristaltismo emenor tempo dehospitalização em relação ao grupo controle. ${ }^{18}$

\section{CONCLUSÃO}

A suplementação alimentar está indicada para pacientes que apresentem depleção do estado nutricional, principalmente naqueles em que se faz necessário o uso de dietas restritivas.

D e todos os aspectos avaliados nos estudos relatados com relação à suplementação nutricional oral de dietas quimicamente definidas pode-se destacar a adequação da oferta de nutrientes, boa aceitação, bem-estar emocional e melhora da ingestão alimentar.

A suplementação com dieta quimicamente definida com baixo teor de resíduos pode fornecer adequado aporte de nutrientes, contribuindo para a melhora do estado nutricional dos pacientes portadores de tumor de canal anal. 


\section{AGRADECIMENTOS}

À Profa Dra Tania Chalhub pela ajuda técnica na elaboração deste trabalho.

\section{REFERÊNCIAS BIBLIOGRÁFICAS}

1. Instituto $\mathrm{N}$ acional de $\mathrm{C}$ âncer (Brasil). 0 problema do câncer no Brasil. 4a ed. Rio de Janeiro: IN CA; 1997.

2. Waitzberg $D$ L. Nutrição enteral eparenteral na prática clínica. 2a ed. Rio deJ aneiro: Atheneu; 1997. p. 451-56.

3. CamposACL, M atiasJEF. N utrição no paciente com câncer. In: M oraes M F. Princípios da cirurgia oncológica. Rio deJ aneiro: Atheneu; 1996. p. 303-23.

4. Carvalho EB, Corrêa M M, Tôrres HOG . Câncer. In: Carvalho EB. M anual de suporte nutricional. Rio de Janeiro: M edsi; 1992. p. 221-32.

5. Dias MCG, Nadalin W, Baxter $Y C$, Faintuch J, Waitzberg DL, M aculevicius J. Acompanhamento nutricional depacientesem radioterapia. Rev Hosp Clin Fac M ed 1996;51:53-9.

6. Carvalho EB, Corrêa M M, Tôrres HOG . N utrição em câncer. In: M urad AM , Katz A. O ncologia bases clínicas do tratamento. Rio de Janeiro: G uanabara Koogan; 1996. p. 121-25.

7. Shils M E, Shike M E. N utritional support of the cancer patient. In: Shils M E, O Ison JA, ShikeM E, et al. In: M odern nutrition in health and disease. Baltimore: Williams \& W ilkins; 1999. p. 1297-322.

8. M anhan LK, Escott-stump S. K rause: alimentos, nutrição e dietoterapia. 9a ed. São Paulo: Roca; 1998. p. 629-55.

9. Hulsew EKW, D eutz NE, Blaauw I, van der $H$ ulst RR, von $M$ eyenfeldt $M M$, Soeters $P B$. Liver protein and glutaminemetabolism during cachexia. Proc N utr Soc 1997;56(2):801-6.

10. Parkinson SA, LewisJ, M orrisR, Allbright A, Plant H, Slevin M L. $O$ ral protein and energy supplements in cancer patients. $\mathrm{H}$ um N utr Appl N utr 1987;41(4):233-43.

11. M atheus $\mathrm{CO}$, Koch $\mathrm{KS}$, N évola AC, et al. Carcinoma epidermóide de canal anal: resultados do tratamento de 46 pacientes. Rev Bras ColoProctol 1997;17:180-5.
12. D avid Filho WJ. Câncer decanal anal. In: Anelli $A$, organizador. M anual prático de condutasem oncologia clínica. São Paulo: LEM AR; 2000.

13. M urad AM , K atz A. O ncologia bases clínicas do tratamento. Rio de Janeiro: Guanabara Koogan; 1996. p. 74-89.

14. CorrêajH S. Tratamento do carcinoma do canal anal com radioterapia, quimioterapiaecirurgia [dissertação]. Rio de Janeiro: 1991.

15. Simon SD. Interações entre radioterapia e quimioterapia. In: Salvajoli JV. Radioterapia em oncologia. Rio deJ aneiro: M edsi; 1999. p. 231-6.

16. Araújo CM M, Pinto LHJ. Princípios da radioterapia. In: M oraes M F. Princípios da cirurgia oncológica. Rio de Janeiro: Atheneu;1996.

17. Salvajoli JV, Weltman E. Princípios de radioterapia. In: M oraes M F. Princípios da cirurgia oncológica. Rio de Janeiro: Atheneu;1996.

18. RumleyTO, Lineaweaver WC, D avisJ M . Low residue nutritional supplementation as an adjunt to mechanical preparation for surgical treatment of the colon. Surg $G$ ynecol 0 bstet 1987;164(4):345-50.

19. Instituto $N$ acional deCâncer (Brasil). Programade nutrição oncológica. Rio deJ aneiro: IN CA; 2000.

20. $\mathrm{N}$ ayel $\mathrm{H}$, El-ghoneimy E, El-haddad S. Impact of nutritional supplementation on treatment delay and morbidity in patients with head and neck tumors treated with irradiation. $\mathrm{N} \mathrm{u}$ trition 1992;8(1):13-8.

21. FranguellaVS, Carvalho L, Silva AF, Ianni G. Avaliação do estado nutricional e conduta dietoterápicaaplicada em pacientescom doenças do aparelho digestivo. M undo Saúde 1997;21(1):36-41.

22. H essov I. D ietoterapia oral e suplementação dietética oral. Rev N utr Enter 1997;12(1):10-3.

23. M cwhinter JP, Pennington CR. A comparison between oral and nasogastric nutritional supplements in malnourished patients. N utrition 1996;12:502-6.

24. $O$ vesen L, Allingstrup L. D ifferent quantities of two commercial liquid diets consumed by weight-losing cancer patients. JPEN 1992;16:275-8.

25. M CC arthy $D$, Weihofen $D$. T he effect of nutritional supplements on food intake in patients undergoing radiotherapy. $\mathrm{O}$ ncol N urs Forum 1999;26(5):897-900. 